\title{
Origem Anômala da Artéria Coronária Esquerda em Tronco Pulmonar (Síndrome de Bland-White-Garland): Diagnóstico por Tomografia Computadorizada Multislice
}

\author{
Márcio Antônio dos Santos¹, Fabiano A. C. Carloto ${ }^{1}$, Arthur Soares Souza Jr. ${ }^{1}$, Moacir F. Godoy ${ }^{1}$
}

$\mathbf{P}$ aciente do sexo feminino, com 56 anos de idade, apresentando quadro de precordialgia relacionada ao esforço físico com progressão para angina de repouso nos últimos meses, acompanhada de dispnéia.

No atendimento na unidade de emergência, a paciente apresentou exame físico com sopro sistólico precordial, pulsos palpáveis e simétricos, e pressão arterial dentro da normalidade. O eletrocardiograma mostrava ritmo sinusal com alteração da repolarização em parede anterior. O ecocardiograma evidenciou função sistólica do ventrículo esquerdo dentro da normalidade e porção proximal da artéria coronária direita dilatada (5 mm), com fluxo turbulento em sua origem. O óstio da artéria coronária esquerda não foi visibilizado.

Durante a internação, foi realizada cintilografia miocárdica, que evidenciou hipoperfusão transitória de grau moderado envolvendo área de moderada/ grande extensão no segmento médio/basal anterior do ventrículo esquerdo. Foi realizada tomografia computadorizada multislice de 16 canais (Figura 1), que evidenciou artéria coronária direita dilatada em sua porção proximal e ausência do óstio da artéria coronária esquerda em seio esquerdo. Havia grande rede de colaterais a partir da artéria coronária direita, perfundindo retrogradamente a artéria coronária esquerda, a qual tinha origem em tronco da artéria pulmonar, confirmando o diagnóstico de origem anômala da artéria

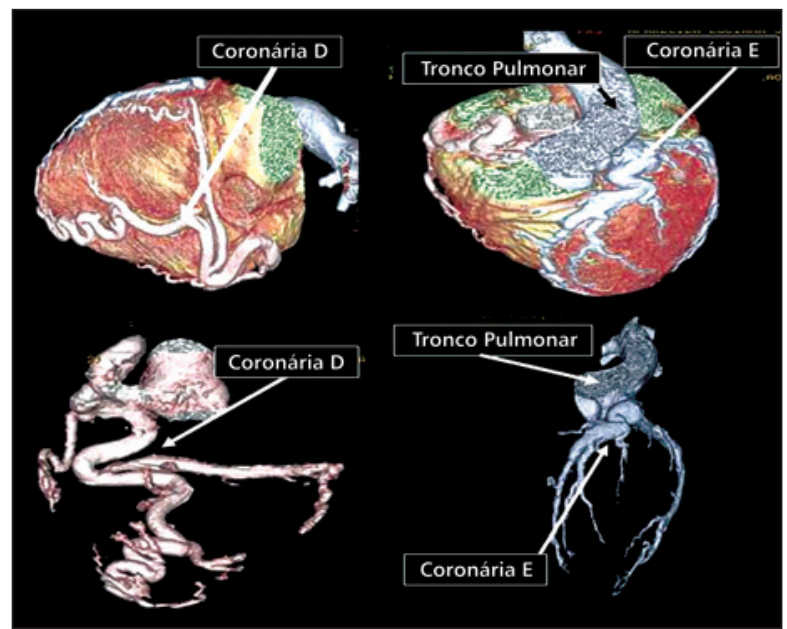

Figura 1 - Imagens de tomografia computadorizada multislice (16 canais), demonstrando origem da coronária direita normal e ausência do óstio esquerdo no seio coronário esquerdo. $\mathrm{D}$ = direita; $\mathrm{E}=$ esquerda.

coronária esquerda em tronco pulmonar tipo adulto (síndrome ALCAPA ou síndrome de Bland-WhiteGarland).

Foi iniciada terapêutica específica para insuficiência coronária e proposto tratamento cirúrgico, recusado pela paciente, sendo mantido tratamento clínico otimizado.

1 Serviço de Hemodinâmica e Cardiologia Intervencionista do Hospital de Base Funfarme/Famerp; Instituto Pró-Cardíaco Rio Preto; Ultra X - Diagnóstico por Imagem - São José do Rio Preto, SP. Correspondência: Márcio Antônio dos Santos. Rua Prof. Carlos Roberto de Oliveira, 67 - Jardim Vivendas - São José do Rio Preto, SP - CEP 15090-380

E-mail: marcioadosantos@terra.com.br

Recebido em: 30/7/2008 • Aceito em: 29/10/2008 